\title{
DIDERIK BATENS \\ Devising the set of abnormalities for a given defeasible rule
}

\author{
Diderik Batens \\ Centre for Logic and Philosophy of Science, Universiteit Gent, \\ Blandijnberg 2, 9000 Gent, Belgium. \\ E-mail: Diderik.Batens@UGent.be
}

\begin{abstract}
Devising adaptive logics usually starts with a set of abnormalities and a deductive logic. Where the adaptive logic is ampliative, the deductive logic is the lower limit logic, the rules of which are unconditionally valid. Where the adaptive logic is corrective, the deductive logic is the upper limit logic, the rules of which are valid in case the premises do not require any abnormalities to be true. In some cases, the idea for devising an adaptive logic does not relate to a set of abnormalities, but to one or more defeasible rules, and perhaps also to one of the deductive logics. Defeasible rules are not universally valid, but are valid in 'normal situations' or for unproblematic parts of premise set. Where the idea is such, the set of abnormalities has to be delineated in view of the rules. The way in which this task may be tackled is by no means obvious and is the main topic studied in the present paper. The outcome is an extremely simple and transparent recipe. It is shown that, except for very special cases, the recipe leads to an adequate result.
\end{abstract}

Keywords: adaptive logics, defeasible reasoning, defeasible rules, conditional derivation, dynamic proofs, abnormalities, falsehood, content guidance

For citation: Batens D. "Devising the set of abnormalities for a given defeasible rule", Logicheskie Issledovaniya / Logical Investigations, 2020, Vol. 26, No. 1, pp. 9-35. DOI: 10.21146/2074-1472-2020-26-1-9-35

\section{Envoi}

This paper is dedicated to the memory of Alexander Karpenko. We got to know each other better when Alexander was responsible for three Moscow institutes in a European project ran by me on behalf of my home university, Ghent, and Salzburg, and Brussels (VUB). I still treasure a booklet with poems by Bielo Cardinal - the White Cardinal, an allusion on Alexander's home country Belarus. I cannot read the poems, let alone understand them. Yet, at 
a dinner party in his home, the poet read some of them to me, and I associated them with the poems of one of my favourite writers in my home tongue, Willem Elsschot, who, apart from a fat volume of novels, left us some twenty impressive poems.

While Sasha declaimed his poems, he became for me the symbol of man reaching for what cannot be attained. That we reach anyway may, more than anything else, makes our lives meaningful. It incites and motivates us to stand by our fellow humans, to build a better world and to create beauty.

\section{Aim Of This Paper}

When awake, humans are in a conscious or semi-conscious state. In that state, their brain activity leads to results of many sorts: perceptions, observations, goals, plans, decisions, etc. Philosophers try to explicate most of that brain activity in terms of reasoning. The bulk of this reasoning is defeasible, not deductive.

Allow me to list ${ }^{1}$ some reasoning forms that are unavoidably defeasible. One first thinks of all kinds of inductive reasoning Batens, 2004, Batens, 2005; Batens, 2011; Batens, Haesaert, 2003; Meheus, 2004], including inductive generalization as well as all predictions derived from the obtained generalizations. There is also abductive reasoning, with its ties to explanations of sorts Batens, 2017; Beirlaen, Aliseda, 2014; Lycke, 2012; Meheus, 2007; Meheus, 2011; Meheus, Batens, 2006; Meheus et al., 2002: Gauderis, Van De Putte, 2012]; but just as well approaches to explanation that do not rely on abduction Batens, 2005: Batens, Meheus, 2001; Weber, De Clercq, 2002, Weber, Van Dyck, 2001]. A very different topic is compatibility, including inconsistent compatibility. Even finding out whether, in general, a predicative set of statements is inconsistent or not, or whether two predicative sets are incompatible with each other or not requires defeasible reasoning [Batens, Meheus, 2000, Meheus, 2003 Meheus, Provijn, 2004. Further examples concern the logic of questions [De Clercq, Verhoeven, 2004, Meheus, 2001], handling deontic conflicts Beirlaen, Straßer, 2013a; Beirlaen, Straßer, 2013b; Goble, 2014; Meheus et al., 2010a; Meheus et al., 2010b; Straßer, 2010; Straßer et al., 2012, Van De Putte et al., in press; Van De Putte, Straßer, 2012] and many more. A whole different family are corrective adaptive logics, like the one for handling inconsistency, started in the 1980s Batens, 1985: Batens, 1986; Batens, 1989 and having resulted in too many papers to refer to in the present context, and those handling ambiguity [Batens, 2002, Vanackere, 1999a; Vanackere, 1999b; Vanackere, 2000; Vanackere, 2001.

\footnotetext{
${ }^{1}$ The interspersed references are incomplete, even with respect to adaptive logics proposed for handling the topics.
} 
The adaptive logics programme is one of the attempts to unify all sensible and useful defeasible reasoning. It is rather easy to devise a manifold of modeltheoretic, procedural, and other systems that define defeasible reasoning forms that no one could possibly unify. All those systems may prove to be interesting and even useful mathematical structures in some more or less distant future. They may also turn out idle tea table talk. So I propose to spend a reasonable part of our efforts to defeasible reasoning forms that are known to be sensible and useful.

Adaptive logics in standard format - see Section 2. - form a unifying structure that is simple and formal. This requires some comments. The relation between the premises and the conclusion of defeasible reasoning is known to be complex. If the explication in terms of adaptive logics is right, as present insights suggest, the complexity of the consequence relation if up to $\Pi_{1}^{1}$-complex Batens et al., 2009; Horsten, Welch, 2007; Odintsov, Speranski, 2012, Odintsov, Speranski, 2013; Verdée, 2009]. Yet the ideas behind the semantics are transparent and unsophisticated. Moreover, there are dynamic proofs. In some cases, the proofs only stabilize at an infinite point - an unavoidable effect of the complexity of the consequence relation. Yet the finite proof stages offer arguably a sensible estimate, in view of the information revealed by the stage, of the result obtained when the proof stabilizes - this is called final derivability. And indeed, proof stages are constructed by simple means. All rules are finitary - unlike for, for example, second order logic. And which lines are IN or OUT in the any given stage of the dynamic proof is decidable. So this basically reflects the human condition: drawing conclusions from the available information is rather unproblematic, but we know this information to be partial and presumably misguided.

I stated that adaptive logics form a formal unifying structure. This means what it always meant: that inferences are correct in view of their form. This does not entail, as some simpletons actually expect, that Uniform Substitution (US) holds. US does not even work for full Classical Logic, CL. ${ }^{2}$ But a different formal criterion strictly obtains; my preferred name for it is bijective uniformity. Technicalities aside, two inference statements $\Gamma \vdash A$ and $\Delta \vdash B$ have the same characteristic form iff each of them can be obtained from the other by systematically replacing a referring term by another referring term of the same sort - an individual constant by an individual constant, a predicate of rank $r$ by a predicate of rank $r$, etc. The result is that, for example, even the propositional inference statements $\neg p \wedge q, p \vee r \vdash r$ and $\neg p \wedge p, p \vee r \vdash r$ do not

\footnotetext{
${ }^{2}$ The closest that comes to it is, to the best of my knowledge, still reported by Witold Pogorzelski and Tadeusz Prucnal Pogorzelski, Prucnal, 1975]; enjoy.
} 
have the same characteristic form because the former cannot be obtained from the latter by any such systematic replacement.

Given the importance of defeasible reasoning, and hence of adaptive logics in standard format as candidates for the unification, it is essential to delineate ways to devise adaptive logics. A general feature about defeasible reasoning is that it capitalizes on the fact that a certain feature or situation is normal in the sense of frequently occurring, whereas abnormal features or situations are exceptional. This leads to the idea to consider certain conclusions are justified in view of the presumed absence of abnormality. Most studied adaptive logics were obtained by first delineating the set of abnormalities, which is characterized by a certain logical form. Thus, even if it turns out that a theory (or data set) requires $\exists x(P x \wedge Q x) \wedge \exists x(P x \wedge \neg Q x)$ to be true, one may still presume that $\exists x(P x \wedge R x) \wedge \exists x(P x \wedge \neg R x)$ is false.

Next, one studies which inferences are defeasibly correct, that is correct in view of the presumed falsehood of certain abnormalities. Clearly, $\exists x(P x \wedge R x) \vdash_{\mathbf{C L}} \forall x(P x \supset R x) \vee(\exists x(P x \wedge R x) \wedge \exists x(P x \wedge \neg R x))$. So if one may, reasoning systematically, consider $\exists x(P x \wedge \neg R x)$ as false, and one knows that $\exists x(P x \wedge R x)$ is true, one may conditionally derive $\forall x(P x \supset R x)$. The justification will go as follows. From the true $\exists x(P x \wedge R x)$ follows $\forall x(P x \supset R x) \vee(\exists x(P x \wedge R x) \wedge \exists x(P x \wedge \neg R x))$. The second disjunct of the conclusion is an abnormality, which we presume to be false and this presumption can be upheld. So, in the light of present insights, $\forall x(P x \supset R x)$ is true. Needless to say, this is merely an intuitive description. The matter will be phrased precisely in Section 2. and references to proofs will be given there.

So the traditional approach was to start from a set of abnormalities and next to study which defeasible inferences are correct if certain abnormalities may be presumed to be false. As becomes clear in the next section, once we know what the abnormalities are, the relevant adaptive logic is easily defined. Adaptive logics consider abnormalities as false in 'normal' situations; as false until and unless proven otherwise.

Often, however, in devising an adaptive logic, one does not know from the beginning which are the abnormalities. Rather, one knows that the reasoning step $\mathrm{A} / \mathrm{B}^{3}$ is correct when 'nothing is wrong', when the situation is normal. Here "normal" points to a further unknown situation, the situation in which the rule $A / B$ is valid.

Concrete examples follow in subsequent sections, but the problem is to collect general insights on the relation between the abnormalities and such a defeasible rule. Does the rule determine the set of abnormalities? Do several

\footnotetext{
${ }^{3}$ Rules are phrased in metalinguistic terms. So I use meta-metalinguistic variables for formulas to describe a rule.
} 
sets of abnormalities make the rule valid as a defeasible rule? If so, what are the effects of different choices?

\section{Preliminaries}

Many introductions to adaptive logics are available - the most recent one is always the best Batens, 2015. So I shall be very brief here. Moreover, the reader may skip this section and look up things in it as she or he needs them to understand subsequent sections.

An adaptive logic, $\mathbf{A L}$, in standard format is a triple:

(i) a lower limit logic LLL: a logic that has static proofs and has a nice semantics; ${ }^{4}$

(ii) a set of abnormalities $\Omega$ : a decidable set of formulas characterized by a (possibly restricted) logical form F; or a union of such sets; ${ }^{5}$

(iii) an adaptive strategy: Reliability or Minimal Abnormality. ${ }^{6}$

The upper limit logic ULL is obtained by extending the lower limit logic LLL with an axiom stating that all abnormalities cause triviality. Where a premise set $\Gamma$ does not require any abnormalities to be true, the $\mathbf{A L}$ consequences of $\Gamma$ provably coincide with its ULL-consequences. One of the effects is that the inconsistency-adaptive consequences of a consistent premise set coincide with the set's CL-consequences.

In a 'Dab-formula' $\operatorname{Dab}(\Delta), \Delta$ is a finite subset of $\Omega$ and $\operatorname{Dab}(\Delta)$ denotes the classical disjunction of the members of $\Delta$. So classical disjunction needs to be present in the language or has to be added. ${ }^{7}$

$\operatorname{Dab}(\Delta)$ is a minimal Dab-consequence of $\Gamma$ iff $\Gamma \vdash_{\mathbf{L L L}} \operatorname{Dab}(\Delta)$ whereas $\Gamma \nvdash_{\mathbf{L L L}} \operatorname{Dab}\left(\Delta^{\prime}\right)$ for any $\Delta^{\prime} \subset \Delta$. A choice set of $\Sigma=\left\{\Delta_{1}, \Delta_{2}, \ldots\right\}$ is a set that contains an element out of each member of $\Sigma$. A minimal choice set of $\Sigma$ is a choice set of $\Sigma$ of which no proper subset is a choice set of $\Sigma$. Where $\operatorname{Dab}\left(\Delta_{1}\right), \operatorname{Dab}\left(\Delta_{2}\right), \ldots$ are the minimal Dab-consequences of $\Gamma, U(\Gamma)=$ $\Delta_{1} \cup \Delta_{2} \cup \ldots$ and $\Phi(\Gamma)$ is the set of minimal choice sets of $\Sigma=\left\{\Delta_{1}, \Delta_{2}, \ldots\right\}$.

\footnotetext{
${ }^{4}$ Read this as a compact Tarski logic with a characteristic semantics. The idea of a nice semantics Verdée, Batens, 2016 is more sophisticated than that of a characteristic semantics and is fascinating in view of its implications for embedding. Unfortunately, explaining it here would require too long a digression.

${ }^{5}$ Where $\mathcal{F}^{a}$ is the set of atomic formulas (those containing no logical symbols other than $=),\left\{A \wedge \neg A \mid A \in \mathcal{F}^{a}\right\}$ is an example of a restricted logical form.

${ }^{6}$ There are the most important strategies.

${ }^{7}$ As Sergei Odintsov and Stanislav Speranski first pointed out Odintsov, Speranski, 2013, an alternative is to phrase adaptive logics in multiple conclusion terms.
} 
Definition 1. A LLL-model $M$ of $\Gamma$ is reliable iff $\operatorname{Ab}(M) \subseteq U(\Gamma)$.

Definition 2. $\Gamma \vDash_{\mathbf{A L}^{r}} A$ iff $A$ is verified by all reliable models of $\Gamma$.

Definition 3. A LLL-model $M$ of $\Gamma$ is minimally abnormal iff there is no LLL-model $M^{\prime}$ of $\Gamma$ such that $\mathrm{Ab}\left(M^{\prime}\right) \subset \mathrm{Ab}(M)$.

Definition 4. $\Gamma \vDash_{\mathbf{A L}^{m}} A$ iff $A$ is verified by all minimally abnormal models of $\Gamma$.

It can be shown that a LLL-model $M$ of $\Gamma$ is minimally abnormal iff $\operatorname{Ab}(M) \in \Phi(\Gamma)$.

Although I started with their semantics, adaptive logics were discovered by reflecting on dynamic proofs - the theorizing on dynamic proof theories came much later [Batens, 2009]. An annotated AL-proof consists of lines that have four elements: a line number, a formula, a justification (at most referring to preceding lines) and a condition. Where

\section{$A \quad \Delta$}

abbreviates that $A$ occurs in the proof as the formula of a line that has $\Delta$ as its condition, the (generic) inference rules are $-\check{V}$ is a classical disjunction:

PREM If $A \in \Gamma$ :

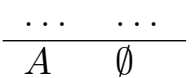

RU

If $A_{1}, \ldots, A_{n} \vdash_{\text {LLL }} B$ :

$A_{1} \quad \Delta_{1}$

\begin{tabular}{ll}
$A_{n}$ & $\Delta_{n}$ \\
\hline$B$ & $\Delta_{1} \cup \ldots \cup \Delta_{n}$
\end{tabular}

$\mathrm{RC}$

$$
\begin{aligned}
& \text { If } A_{1}, \ldots, A_{n} \vdash_{\mathbf{L L L}} B \check{\mathrm{V}} \mathrm{Dab}(\Theta) \quad A_{1} \quad \Delta_{1} \\
& \text {... } \ldots \\
& \begin{array}{ll}
A_{n} & \Delta_{n} \\
\hline B & \Delta_{1} \cup \ldots \cup \Delta_{n} \cup \Theta
\end{array}
\end{aligned}
$$

A proof stage is a list of lines obtained by applications of the generic rules PREM, RU and RC. Let the empty list be stage 0 . Where $s$ is a stage, $s^{\prime}$ is an extension of $\mathrm{s}$ iff all lines that occur in $\mathrm{s}$ occur in the same order in $\mathrm{s}^{\prime}$. A (dynamic) proof is a chain of stages. That $A$ is derivable on the condition $\Delta$ may be interpreted as: it follows from the premise set that $A$ or one of the members of $\Delta$ is true. Because the members of $\Delta$, which are abnormalities, are presumed to be false, $A$ is considered as derived, unless and until it shows that 
the presumption cannot be upheld. The precise meaning of "cannot be upheld" depends on the strategy, which determines the marking definition (see below) and hence determines which lines are marked at a stage. If a line is marked at a stage, its formula is considered as not derived at that stage.

$\operatorname{Dab}(\Delta)$ is a minimal Dab-formula at stage s of an $\mathbf{A L}$-proof iff $\operatorname{Dab}(\Delta)$ was derived at $\mathrm{s}$ on the condition $\emptyset$ whereas for no $\Delta^{\prime} \subset \Delta$ was $\operatorname{Dab}\left(\Delta^{\prime}\right)$ derived on the condition $\emptyset$. Where $\operatorname{Dab}\left(\Delta_{1}\right), \ldots, \operatorname{Dab}\left(\Delta_{n}\right)$ are the minimal Dab-formulas at stage $\mathrm{s}$ of a proof from $\Gamma, U_{s}(\Gamma)=\Delta_{1} \cup \ldots \cup \Delta_{n}$ and $\Phi_{s}(\Gamma)$ is the set of minimal choice sets of $\left\{\Delta_{1}, \ldots, \Delta_{n}\right\}$.

Definition 5. Marking for Reliability: Line $l$ is marked at stage s iff, where $\Delta$ is its condition, $\Delta \cap U_{s}(\Gamma) \neq \emptyset$.

Definition 6. Marking for Minimal Abnormality: Line $l$ is marked at stage s iff, where $A$ is derived on the condition $\Delta$ on line $l$, (i) there is no $\varphi \in \Phi_{s}(\Gamma)$ such that $\varphi \cap \Delta=\emptyset$, or (ii) for some $\varphi \in \Phi_{s}(\Gamma)$, there is no line on which $A$ is derived on a condition $\Theta$ for which $\varphi \cap \Theta=\emptyset$.

Let me rephrase this: where $A$ is derived on the condition $\Delta$ on line $l$, line $l$ is unmarked at stage s iff (i) there is a $\varphi \in \Phi_{s}(\Gamma)$ for which $\varphi \cap \Delta=\emptyset$ and (ii) for every $\varphi \in \Phi_{s}(\Gamma)$, there is a line at which $A$ is derived on a condition $\Theta$ for which $\varphi \cap \Theta=\emptyset$.

A formula $A$ is derived at stage s from $\Gamma$ iff it is the formula of a line that is unmarked in s. Marks may come and go as the proof proceeds. Yet there also is a stable notion of derivability, called final derivability.

Definition 7. $A$ is finally derived from $\Gamma$ on line $l$ of stage $\mathrm{s}$ iff (i) $A$ is the second element of line $l$, (ii) line $l$ is not marked at stage $\mathrm{s}$, and (iii) every extension of the stage in which line $l$ is marked may be further extended in such a way that line $l$ is unmarked.

Definition 8. $\Gamma \vdash_{\mathbf{A L}} A$ ( $A$ is finally $\mathbf{A L}$-derivable from $\Gamma$ ) iff $A$ is finally derived on a line of a proof stage from $\Gamma$.

There are three comments in conclusion of the preliminaries. First, adaptive logics are not competitors of deductive logics, but means to arrive at formal characterizations of methods. Next, one typically needs adaptive logics (and, more generally, defeasible reasoning) when a positive test is absent. Consider any of the examples mentioned before. At the predicative level, the consequence set of the adaptive logics is not semi-recursive. The final comment is that adaptive logics have an impressive metatheory which required the development of novel proof methods. The metatheory includes Soundness and Completeness proofs, but also the proofs of many features that are entirely 
foreign to deductive logics. I refer to Batens, 2007 for the metatheory and to [Batens, 2015] for a revised formulation of the theorems, often leaving the straightforward reformulation of the proofs to the reader.

\section{The Problem}

Consider a defeasible rule $A_{1}, \ldots, A_{n} / B$ that we consider as valid in normal situations. In rather exceptional cases we have no precise idea of the lower limit logic LLL, but let us neglect that problem and suppose that the strategy as well as LLL are given. ${ }^{8}$ The task is to find the form of a formula $C$ that may serve as the abnormality for the rule, viz. such that $A_{1}, \ldots, A_{n} \vdash_{\text {LLL }} B \vee \bar{C}$. Once this $C$ is found, the corresponding conditional rule $C R$ will be: "from $\mathrm{A}_{1}, \ldots, \mathrm{A}_{n}$ on the condition $\Delta$ to derive $\mathrm{B}$ on the condition $\Delta \cup\{\mathrm{C}\}$ ".

The reader may wonder whether a single abnormality $C$ is introduced rather than a Dab-formula, as was suggested by the way the generic rule RC was phrased in the previous section. This is an interesting point. Let us leave open whether $C$ will be the form of the abnormalities or whether $C$ may indeed be itself a disjunction of abnormalities. Let us also leave open whether the problem is to find a unique $C$ or several - the latter case refers to the second alternative in the description of the set of abnormalities $\Omega$ : "or a union of such sets". The first sentence of the present paragraph moreover reminds us of an important matter. We want to delineate $\Omega$ in function of the defeasible rule $A_{1}, \ldots, A_{n} / B$. Yet, we are after an adaptive logic in standard format. In other words, the generic rule $\mathrm{RC}$ will by no means be restricted to the defeasible rule $\mathrm{A}_{1}, \ldots, \mathrm{A}_{n} / \mathrm{B}$. The generic rule $\mathrm{RC}$ will solely depend on $\mathbf{L L L}$ and $\Omega$ as is obvious from Section 2. We shall see that this consideration will play an important role in subsequent pages.

Consider some examples of defeasible rules in the domain of inductive generalization. There are many adaptive logics in that domain. Each of them characterizes a way to defeasibly infer generalizations. A generalization is a formula $\forall x A(x)$ in which $A(x)$ is a truth function of literals in which no individual constants occur. ${ }^{9}$ One of the logics allows one to introduce generalizations as Popperian hypotheses, the defeasible rule then becoming $-/ \forall x A(x)-$ given whatever, one may conditionally introduce a generalization. Other such logics require an instance and hence need a defeasible rule $A(\alpha) / \forall x A(x)$, in which $\alpha$ may be any individual constant. Still, other logics require a 'positive instance'

\footnotetext{
${ }^{8}$ When we are after an ampliative adaptive logic, LLL will be the deductive logic we consider suitable in the given context. For many this will be CL when the context concerns empirical or classical mathematical theories.

${ }^{9}$ The precise formulation was published elsewhere Batens, 2011, but is not terribly important in the present context.
} 
as in $B(\alpha) \wedge C(\alpha) / \forall x(B(x) \supset C(x))$. In all of these $A$ and $C$ are disjunctions of one or more literals and $B$ is a conjunction of literals - conjunctions of two generalizations are derived by RU from generalizations derived by RC. So, for each such defeasible rule, the task is to pinpoint an abnormality, which then will determine the set of abnormalities $\Omega$ for that logic.

The sets of abnormalities for those inductive generalization rules were delineated a long time ago by tinkering. This was not difficult and they agree nicely with the recipe that will be presented in the present paper. This is a good reason to consider a different type of adaptive logics.

It is desirable to refer to a case where the matter becomes slightly more difficult as well as slightly more interesting. While working on adaptive set theories [Batens, 2019], I came about a case that I never met before. That we are dealing with a corrective adaptive logic is a difference with the logics from the previous paragraphs. Yet, something is more important. The lower limit logic of the set theories is the paraconsistent CLuNs, which is specified below, and the strategy is Minimal Abnormality. The well-studied inconsistencyadaptive logic $\mathbf{C L u N s}{ }^{m}$ is obtained by specifying the set of abnormalities as $\left\{\mathrm{Q}(A \wedge \neg A) \mid A \in \mathcal{F}^{a}\right\}$, in which $\mathcal{F}^{a}$ is the set of (open and closed) atomic formulas and $\mathrm{Q}(A)$ is $(A)$ preceded by a quantifier over every formula free in $A$. I give this set a specific name for future reference. It turns out that certain premise sets require a different adaptive logic, one that has a more embracing set of abnormalities and hence assigns a richer consequence set to the premise sets.

While adaptive logics were originally devised as ways to formally characterize methods, it turned out that they may also be profitably invoked to characterize complex theories - viz. theories that are not semi-recursive. Partly relying on work by others, I made attempts to devise adaptive theories for Peano Arithmetic and for Frege's notion of a set. It is the latter that led to the case I now shall outline. I'll just mention some ideas, as the paper will soon be available in print. However, there are some details I have to report explicitly in order to clarify the problem. Readers who are in a hurry may skip to the beginning of Section 4 . and return here later if they get interested in the significant example.

As Frege's notion of a set makes inconsistent sets unavoidable, the lower limit logic of the adaptive logic needs to be paraconsistent. For reasons not discussed here, I choose the (very popular) paraconsistent logic (which I prefer to call) CLuNs Batens, Clercq, 2004. Apart from negation, all logical symbols 
are exactly as in $\mathbf{C L}$ and RoI (Replacement of Identicals) holds unrestrictedly. ${ }^{10}$ The negation $\neg$ is strictly paraconsistent ${ }^{11}$ and reduces complex negations to simpler ones in the usual way: $\neg \neg A \equiv A, \neg(A \wedge B) \equiv(\neg A \vee \neg B), \ldots$ and $\neg \exists x A \equiv \forall x \neg A$.

The set theory obtained by CLuNs from (a version of) the Fregean axiom schema Abs and axiom Ext will be called PFS (paraconsistent Fregean set theory). ${ }^{12}$ Obviously, one wants to move from the paraconsistent theory to an adaptive one, call it AFS. While one has unavoidably to allow for some inconsistent sets - sets of which some members are also non-members - one wants that sets are only inconsistent when this is unavoidable, and one wants even inconsistent sets to behave as consistently as possible. For example, one wants $\emptyset$ to be consistent and, while the Russell set $R$ is unavoidably inconsistent, one wants $\emptyset \notin R$ in view of $\emptyset \notin \emptyset$ and one does not want $\emptyset \in R$.

Just like the language of most mathematical theories, the language of set theory is extremely simple. Apart from the logical symbols and the variables of the standard predicative language, it has one binary predicate $\in$ and often abstracting terms of the form $\{\alpha \mid A(\alpha)\}$. Where the underlying logic is CLuNs, some formulas of this language express triviality, ${ }^{13}$ for example $\forall x \forall y(x=y \wedge x \neq y \wedge x \in y \wedge x \notin y)$, which I shall abbreviate as $\perp .{ }^{14}$ Literally every formula of the set theoretical language is CLuNs-derivable from this (as well as from some other formulas). ${ }^{15}$ Given that material implication is present

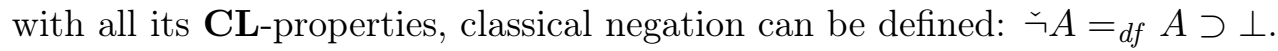

The presence of classical negation has the unexpected consequence that the

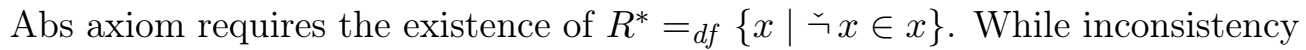
results, $R^{*} \in R^{*} \wedge R^{*} \notin R^{*}$, it is provable that $R^{*} \in R^{*} \wedge \check{\neg} R^{*} \in R^{*}$ is not derivable and that the inconsistency-adaptive theory is non-trivial, just like the paraconsistent theory. Yet, the fact that $R^{*} \in R^{*}$ is a theorem of the

\footnotetext{
${ }^{10}$ RoI: $a=b \supset\left(A \equiv A_{a / b}\right)$ in which $A_{a / b}$ is the result of replacing in $A$ an occurrence of $a$ by $b$ or vice versa. In some paraconsistent logics, RoI does not hold within the scope of a negation.

${ }^{11}$ A negation $\neg$ is paraconsistent iff $A, \neg A \vdash B$ does not hold for all $A$ and $B$; it is strictly paraconsistent iff there is no $A$ such that $A, \neg A \vdash B$ holds for all $B$.

${ }^{12}$ Within CLuNs there are three different implications that coincide in CL: $A \supset B$ is detachable but not contraposable, $A \sqsupset B=_{d f} \neg A \vee B$ is not detachable but contraposable, $A \rightarrow B=_{d f}(A \supset B) \wedge(\neg B \supset \neg A)$ is both detachable and contraposable; similarly, there are 16 different equivalences that coincide in CL. So choices have to be made as one moves from Frege's trivial theory to the provably non-trivial CLuNs-theory PFS.

${ }^{13}$ Several other paraconsistent logics have the same property.

${ }^{14}$ The abbreviations $t_{1} \neq t_{2}={ }_{d f} \neg t_{1}=t_{2}$ and $t_{1} \notin t_{2}={ }_{d f} \neg t_{1} \in t_{2}$ occur for readability.

${ }^{15}$ The formula does not express triviality in some extensions of the language of set theory. So it is a remarkable case of expressing local triviality, a feature that also occurs in other mathematical theories - I shall soon publish a brief study of the remarkable phenomenon and its epistemic potential.
} 
paraconsistent theory, and hence also of the adaptive one, reveals a perhaps unpleasant but interesting phenomenon: $R^{*}$ has members that do not fulfil the touchstone of $R^{*}$. Indeed, $R^{*} \in R^{*}$ is a theorem of the paraconsistent theory,

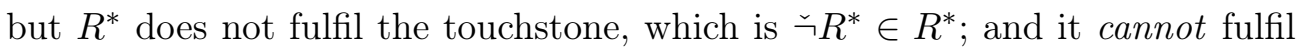
the touchstone - the theory is non-trivial. I shall say that $R^{*}$ is clean iff $\forall y(y \in\{x \mid A(x)\} \leftrightarrow A(y))$, in which $A \leftrightarrow B={ }_{d f}(A \equiv B) \wedge(\neg B \equiv \neg A)$. It turns out that both $\forall y(A(y) \supset y \in\{x \mid A(x)\})$ and $\forall y(\neg A(y) \supset y \notin\{x \mid$ $A(x)\})$ can be required to hold, but not their converses, precisely because some sets, for example $R^{*}$ are unavoidably unclean. The converses have to read $\forall y(y \in\{x \mid A(x)\} \sqsupset A(y))$ and $\forall y(y \notin\{x \mid A(x)\} \sqsupset \neg A(y))$ - remember that $\sqsupset$ is not detachable. ${ }^{16}$

This situation reveals a problem that requires a solution. Indeed, $R={ }_{d f}$ $\{x \mid x \notin x\}$ is inconsistent but there is no need for it to be unclean. Let $\Omega_{1}={ }_{d f}\left\{\mathrm{Q}(A \wedge \neg A) \mid A \in \mathcal{F}^{a}\right\}$, in which $\mathcal{F}^{a}$ is the set of (open and closed) atomic formulas and $\mathrm{Q}(A)$ is $(A)$ preceded by a quantifier over every formula free in $A$. Consider a PFS-model $M$ that is minimally abnormal with respect to $\Omega_{1}$. Obviously, the domain $D$ of $M$ is uncountable ${ }^{17}$ whence some elements of $D$ have no name - are not named by an abstracting term. It turns out that some sets are clean in some PFS-models that are minimally abnormal with respect to $\Omega_{1}$, but are unclean in other PFS-models that are also minimally abnormal with respect to $\Omega_{1}$. A typical example is precisely $R$. Consider a minimally abnormal PFS-model $M_{1}$ in which $R$ is clean and consider an element $o \in D$ that stands in the $\in$-relation to $R$ and not also in the $\notin$-relation to $R$ - technically this will be expressed for example by $\langle o, v(R)\rangle \in v^{T}(\in){ }^{18}$ Next consider a model $M_{2}$ that is exactly like $M_{1}$ except in that $o$ is not only a member but also a non-member of $R$. So, in $M_{2}$, the set $R$ is unclean as well as inconsistent. Yet, given that no individual constant of the language refers to $o$, the inconsistency can only be stated as $\exists x(x \in R \wedge x \notin R)$. But this formula is also verified by $M_{1}$, because all PFS-models verify $R \in R \wedge R \notin R$. So $R$ is clean in $M_{1}$ and is unclean in $M_{2}$, but both are minimally abnormal and actually $\mathrm{Ab}\left(M_{1}\right)=\mathrm{Ab}\left(M_{2}\right)$. This is not as we want it. The axioms do not require $R$ to be unclean. They do not even require that $R$ is a member

\footnotetext{
${ }^{16}$ It is not really essential to this paper, but Abs comes to the conjunction of the four implications mentioned in the text, two detachable ones and two non-detachable ones.

${ }^{17}$ Many uncountable ZF-hierarchies can be defined in exactly the same way in PFS and if their members were countable in PFS, then they would be inconsistent. It can be argued that they are consistent in minimally abnormal models of PFS if they are consistent in $\mathbf{Z F}$.

${ }^{18}$ In this semantic style, the extension of a predicate $\pi$ of rank $n$ is a triple $\left\langle\Sigma_{1}, \Sigma_{2}, \Sigma_{3}\right\rangle$ with $\Sigma_{1}, \Sigma_{2}, \Sigma_{3} \supseteq D^{n}$ and $\Sigma_{1} \cup \Sigma_{2} \cup \Sigma_{3}=D^{n}$. Next, for convenience, the assignment function $v(\pi)$ is seen as three functions: $v^{T}(\pi)=\Sigma_{1}, v^{B}(\pi)=\Sigma_{2}$ and $v^{F}(\pi)=\Sigma_{3}$.
} 
of a minimal set of sets one of which is bound to be unclean. So $R$ should be clean. ${ }^{19}$

What does this come to? We want the non-detachable $\forall y(y \in\{x \mid A(x)\} \sqsupset$ $A(y))$ to have the strength of the detachable $\forall y(y \in\{x \mid A(x)\} \supset A(y))$ as much as possible; and similarly for upgrading $\forall y(y \notin\{x \mid A(x)\} \sqsupset \neg A(y))$ to $\forall y(y \notin\{x \mid A(x)\} \supset \neg A(y))$. Obviously, we do not want to interpret all expressions $A \sqsupset B$ as much as possible as $A \supset B$. We only want instances of schema Abs to be as much as possible detachable in all directions. Abs was intended originally as detachable in all directions. This cannot be realized completely because Frege's notion of the extension of a predicate turned out inconsistent. Nevertheless, the original intention can be realized as much as possible. But this cannot be done by minimizing inconsistencies: remember that $M_{1}$ and $M_{2}$ verify the same members of $\Omega_{1}$. We have a case where, for ordered pairs $\langle A, B\rangle$ of certain forms, we want to derive $A \supset B$ from $A \sqsupset B$ on a certain condition. The task is to find the condition.

\section{Solving the Problem}

Let us concentrate first on rules with one local premise. The task then is, starting from the defeasible rule $A / B$, to find a condition or conditions $C$ such that the three following hold:

\section{$\mathrm{A} \vdash_{\mathbf{L L L}} \mathrm{B} \check{V} \mathrm{C}$ and $\mathrm{A} \nvdash_{\mathbf{L L L}} \mathrm{B}$ and $\mathrm{A} \nvdash_{\mathbf{L L L}} \mathrm{C}$}

If $A \vdash_{\mathbf{L L L}} \mathrm{B}$, then $\mathrm{A} / \mathrm{B}$ is $\mathbf{L L L}$-valid and not a defeasible rule. If $\mathrm{A} \vdash_{\mathbf{L L L}} \mathrm{C}$, then the condition $C$ causes the rule $A / B$ to have no sensible applications. Remember indeed that, as stated in Section 3. the idea is to obtain the following particular conditional rule: "from $\mathrm{A}$ on the condition $\Delta$ to derive $\mathrm{B}$ on the condition $\Delta \cup\{C\} "$. If the defeasible rule $A / B$ is to have sensible applications, there must be a premise set $\Gamma$ and a (empty or non-empty) $\Delta \subset \Omega$ such that (i) $A$ is finally derivable from $\Gamma$ on the condition $\Delta$ and (ii) $\mathrm{B}$ is finally derivable from $\Gamma$ on the condition $\Delta \cup\{C\}$. In view of (i), $\Gamma \vdash_{\text {LLL }} A \vee \operatorname{Dab}(\Delta)$ and there is a $\varphi \in \Phi(\Gamma)$ such that $\Delta \cap \varphi=\emptyset$. In view of (ii), $\Gamma \vdash_{\text {LLL }} \operatorname{BV̌Dab}(\Delta \cup\{C\})$ and there is a $\varphi \in \Phi(\Gamma)$ such that $(\Delta \cup\{C\}) \cap \varphi=\emptyset$. The latter is impossible because, if $A \vdash_{\text {LLL }} C$, then $\Gamma \vdash_{\mathbf{L L L}} \operatorname{AV} \operatorname{Dab}(\Delta)$ is a sufficient condition for $\Gamma \vdash_{\mathbf{L L L}} \mathrm{CV̌ \operatorname {Dab }}(\Delta)$, which is $\Gamma \vdash_{\mathbf{L L L}} \operatorname{Dab}(\Delta \cup\{\mathrm{C}\})$. Whether $\operatorname{Dab}(\Delta \cup\{\mathrm{C}\})$ is a minimal Dab-consequence of $\Gamma$ or not, every $\varphi \in \Phi(\Gamma)$ contains at least one

\footnotetext{
${ }^{19}$ The difference between $M_{1}$ and $M_{2}$ cannot be expressed in the language by a formula stating a contradiction, whether plain or quantified. Does this mean that the difference between $M_{1}$ and $M_{2}$ cannot be expressed? By no means. We just need different abnormalities.
} 
member of $\operatorname{Dab}(\Delta \cup\{C\})$. But then the line at which $B$ is derived by $A / B$ is always marked. This ends the justification of the requirements in (1). ${ }^{20}$

Let us move to a concrete case, viz. the defeasible rule

$$
A \sqsupset B / A \supset B \text {. }
$$

We are looking for one or more conditions such that the three derivability statements in (11) are fulfilled. I shall first consider the problem in the context

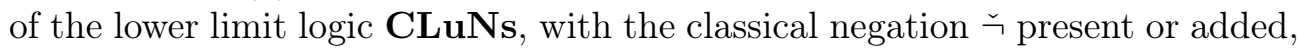
and the Minimal Abnormality strategy, but neglecting for the moment that the problem arose in connection with the set theory AFS.

In the previous section, (2) was considered in a situation in which the abnormalities were contradictions, as is usual for inconsistency-adaptive logics. Some people will keep repeating that abnormalities of the form $A \wedge \neg A$ justify the defeasible rule (2). Indeed,

$$
A \sqsupset B \vdash_{\mathrm{CLuNs}}(A \supset B) \check{V}(A \wedge \neg A)
$$

holds. Or, even more explicitly in view of CLuNs-equivalences,

$$
\neg A \vee B \vdash_{\mathrm{CLuNs}}(\neg A \vee B) \check{\vee}(A \wedge \neg A) .
$$

However, and as already explained in Section 3. this is not the point. ${ }^{21}$ The point is that, for specific ordered pairs $\langle A, B\rangle$, we want (2) to be applied even if $A \wedge \neg A$ is true. ${ }^{22}$

We are in search of conditions $C$ that fulfill (1) and, by their forms, determine a set $\Omega$ that, together with the lower limit logic CLuNs and the Minimal Abnormality strategy, defines an adequate adaptive logic. We need a $C$ such that $A \vdash_{\text {CLuNs }} B \breve{V}$. Given that $\mathbf{C L u N s}$ has a nice semantics and given Soundness and Completeness, $A \vdash_{\mathrm{CLuNs}} \mathrm{B} \check{\mathrm{C} C}$ is equivalent to $\mathrm{A} \check{\wedge} \check{\neg} \vdash_{\mathrm{CLuNs}} \mathrm{C}$. The strongest such $C$ is obviously $A \check{\neg}$ B itself and every such $C$ is a CLuNsconsequence of $\mathrm{A} \check{\wedge} \mathrm{A}$.

Let us apply this at once to the defeasible rule (2). The strongest condition $\mathrm{C}$ is $(A \sqsupset B) \wedge \neg(A \supset B)$, which is CLuNs-equivalent to $(A \wedge \neg A \wedge \neg B)-$ as the conjunction is classical in CLuNs, there is no need to write $\check{\wedge}$. So the defeasible rule phrased with its strongest condition reads: "from $A$ on the condition $\Delta$ to derive $B$ on the condition $\Delta \cup\{A \wedge \neg A \wedge \check{\neg} B\}$ ". Actually, the

\footnotetext{
${ }^{20}$ The justification considers only Minimal Abnormality. Where Reliability is the strategy, the justification is much simpler and left to the reader.

${ }^{21}$ Moreover and concerning AFS, every unclean set is unavoidably inconsistent: if $t \in\{x \mid$ $A(x)\}$ but $\neg A(t)$, then $t \notin\{x \mid A(x)\}$. However, this too is not the point.

${ }^{22}$ In AFS we want all sets to be as clean as possible, even inconsistent sets.
} 
logic CLuNs requires ${ }^{23}$ that the set of abnormalities contains all formulas of the form $A \wedge \neg A \wedge \neg B$ in which $A$ is an atomic formula and $B$ is a literal. ${ }^{24}$

Next, consider weaker conditions $C$ for the defeasible rule (2). Let us have a systematic look at 'parts' of $A \wedge \neg A \wedge \neg B$. The idea is not to find a $C$ that in itself gives us all we want, but to find conditions $C$ that are acceptable, possibly in the presence of other conditions. I neglect the fact that the local premise may come on a condition itself; by now, the reader will have understood the resulting complication. To the left is the CLuNs-inference, to the right the effect on a dynamic proof.

$$
\frac{A \sqsupset B}{(A \supset B) \vee C} \quad \frac{A \sqsupset B}{A \supset B \quad\{\mathrm{C}\}}
$$

Let us consider the possibilities systematically.

(i) We know already that the strongest $\mathrm{C}$ is $A \wedge \neg A \wedge \check{\neg}$.

(ii) That $\mathrm{C}$ is $A \wedge \neg A$ is all right provided one also wants all conditional inferences that then are correct in view of the standard format, specifically RC. An example is the effect of $A \supset B, \neg B \vdash_{\mathrm{CLuNs}} \neg A \vee(B \wedge \neg B)$ : from $A \supset B$ and $\neg B$ to derive $\neg A$ on the condition $\{B \wedge \neg B\}$. So what this comes to is that the choice $A \wedge \neg A$ is all right in case one agrees that $\neg A$

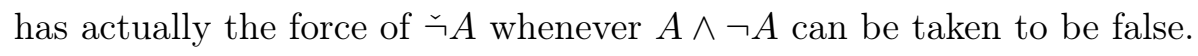

(iii) That $C$ is $A \wedge \neg B$ is not acceptable. Indeed, this condition is simply

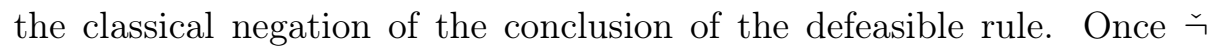
is added to CLuNs, $(A \supset B) \vee(A \wedge \check{\neg} B)$ is a CLuNs-theorem. So if $A \wedge \check{\neg} B$ is an abnormality, possibly with $A$ restricted to atomic formulas

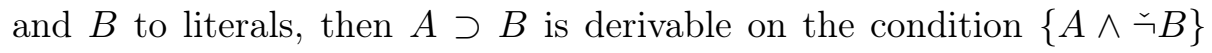
from any premise set. Unlike what the reader might expect, this would not cause premise sets to have trivial consequence sets; most conditional lines would be marked. Yet, there is no sensible idea behind this choice of an abnormality and the choice does not seem to lead to anything sensible. Nevertheless, I shall return to this choice below.

\footnotetext{
${ }^{23}$ The requirement is related to the avoidance of so-called flip-flop adaptive logics, which are only desirable for specific applications Batens, 2007. The point need not further concern us here.

${ }^{24}$ The set of literals is the set of non-equivalent formulas in which occurs an atomic formula

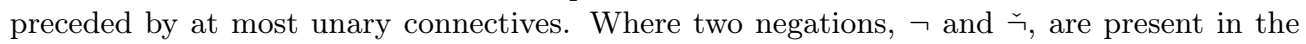
language of CLuNs, the notion of a literal is a trifle more sophisticated than in CL. While

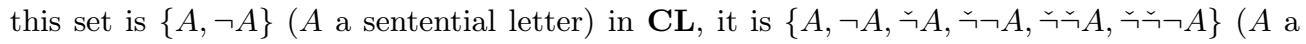
sentential letter) in CLuNs.
} 
(iv) Somewhat unexpectedly, it seems all right at first sight to choose $\neg A \wedge \check{\neg} B$ as $C$. Indeed, when one concentrates on the defeasible rule we are considering here, the choice seems unobjectionable, both in case $\neg A \wedge \neg B$ is true and in case it is false. In the latter case, for example, we obtain: if $\neg A$ is false, the premise warrants that $B$ is true; if $\neg B$ is false, then $B$ is also true. As $B$ is true, so is $A \supset B$. And yet this choice has consequences we do not want. As $\neg A \vdash_{\mathrm{CLuNs}} B \vee(\neg A \wedge \neg B)$, the choice would justify that one would derive an arbitrary $B$ from $\neg A$ on the sole condition that $\neg A \wedge \neg B$ can be taken to be false. As in the previous case, triviality would not result ${ }^{25}$ but there is no idea behind this way of proceeding and nothing sensible is expected to result.

(v) To choose $A$ or $\neg A$ or $B$ as $C$ is obviously unacceptable. That literals would be abnormalities, would result in all kinds of turmoil, but in nothing sensible.

(vi) I promised to return to (iii). Choosing $(A \sqsupset B) \wedge(A \wedge \check{\neg} B)$ as $\mathrm{C}$ prevents one to introduce detachable implications from the blue. Moreover, these abnormalities nicely express that the premise is true and the desired conclusion false. However, nothing new is arrived at along this road. The chosen abnormality is CLuNs-equivalent to $A \wedge \neg A \wedge \neg B$, which is the abnormality considered in (i).

No other choices are worth commenting upon. Yet it is still interesting to consider combinations, viz. that formulas of different forms are counted as abnormalities, for example $A \wedge \neg A$ and $A \wedge \neg A \wedge \neg B$. Neglecting some complications, a line is unmarked and its formula is not considered as derived iff its condition can be considered to be false. Suppose that $A \wedge \neg A$ cannot be considered as false. Then it is nevertheless possible that $A \wedge \neg A \wedge \breve{\neg} B$ can be considered as false: if $A$ and $\neg A$ are both true, but $\neg B$ is false, then the conjunction of the three formulas is false. ${ }^{26}$ So allowing for abnormalities of both forms has the following effect - I keep restricting attention to crucial insights. On the one hand, including formulas $A \wedge \neg A$ in the set of abnormalities has the effect that a lot of further conditional inferences become valid, as was explained in (ii). On the other hand, even if $A \supset B$ cannot be seen as derived on the condition $A \wedge \neg A$ because this condition cannot be considered as false,

\footnotetext{
${ }^{25}$ Adaptive logics in standard format have the Strong Reassurance property (also called Stopperedness or Smoothness): if a premise set has LLL-models, then it has minimally abnormal models. Proofs were published long ago Batens, 2000; Batens, 2007.

${ }^{26}$ Spelling the matter out in a precise way for Reliability and (especially) for Minimal Abnormality is much more complicated, but the crucial insight is the one stated in the text.
} 
it is possible that $A \supset B$ can be seen as derived on the condition $A \wedge \neg A \wedge \neg B$

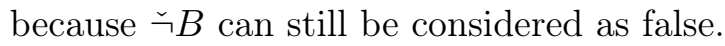

The matter seems clarified, but there are still two little problems. I comment on them in order to illustrate the complications involved in the systematisation of defeasible reasoning. The easier problem is this: $A \wedge \neg A \wedge \neg A$ obviously has the form of $A \wedge \neg A \wedge \neg B$ and is always false. ${ }^{27}$ That looks frightening. As the line will never be marked, such an adaptive logic seems to extend the lower limit logic with non-defeasible steps. However, this is a pseudo-problem. Whenever $\mathrm{A} \vdash_{\mathbf{C L u N s}} \mathrm{B} \vee \mathrm{C}$ and $\mathrm{C} \in \Omega$ is logically false, then $\mathrm{A} \vdash_{\mathbf{C L u N s}} \mathrm{B}$. So logically impossible abnormalities are harmless; they are obviously also useless.

The second problem is more interesting: if formulas of the form $A \wedge \neg A \wedge \neg B$ are abnormalities, does it then even make a difference whether formulas of the form $A \wedge \neg A$ are also abnormalities? While $\neg A \nvdash_{\mathbf{C L u N s}} \sqsupset A \vee(A \wedge \neg A \wedge \neg B)$,

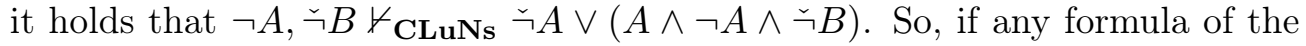

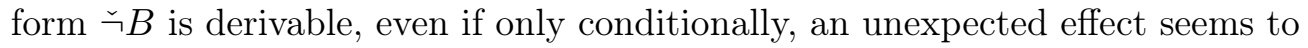
follow. Let me show this by presenting a little proof.

51

52

53 $\check{\neg} s$

$\neg p$

$\check{\sim} p$

$\vdots \quad \vdots$

․ $\Delta$

PREM $\quad \emptyset$

51,$52 ; \mathrm{RC} \quad \Delta \cup\{p \wedge \neg p \wedge \neg s\}$

Supposing that line 51 is unmarked, line 53 will be unmarked just in case

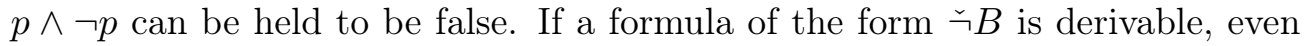
conditionally, from the premises, then abnormalities of the form $A \wedge \neg A$ are redundant.

The matter becomes less surprising if one realizes that conditional transition from $\neg p$ to $\neg p$ may be realized in a way that seems unobjectionable. Recall that $A \sqsupset B$ abbreviates $\neg A \vee B$.

$$
\check{\neg} s
$$$$
\neg p
$$$$
p \sqsupset s
$$$$
p \supset s
$$$$
\neg p
$$

:

...

PREM

52; RU

53; RC

51, 54; RU
$\Delta$

$\emptyset$

$\emptyset$

What happens here is that we apply the defeasible rule (2) at line 54 and next apply Modus Tollens - this is correct as $\supset$ is detachable and $\check{\neg}$ is classical negation.

\footnotetext{
${ }^{27}$ Many will not care about the detail, but it is more correct to say that $A \wedge \neg A \wedge \leftrightharpoons A$ has no non-trivial models - in some semantic styles no models, in others only the trivial model.
} 
If a bottom constant $\perp$ is present in the applied version of CLuNs, the matter is even easier.

$\begin{array}{llll}1 & \neg p & \text { PREM } & \emptyset \\ 2 & \check{\neg} & 1 ; \mathrm{RC} & \{p \wedge \neg p \wedge \check{\neg} \perp\}\end{array}$

Here $\vdash_{\text {CLuNs }} \breve{\perp}$ whence $p \wedge \neg p \wedge \check{\neg} \perp$ is CLuNs-equivalent to $p \wedge \neg p$.

After discussing (2) independently of the set theoretical context, let us now return to the problem in AFS. Two insights are important. (i) If, reasoning systematically, it is possible to consider $A \wedge \neg A$ as false, then it is possible to consider $A \wedge \neg A \wedge \neg B$ as false, but not conversely. So, given a premise set $\Gamma$, the more complex condition allows for final consequences of $\Gamma$ that are not final consequences if all abnormalities have the form $A \wedge \neg A$. (ii) As noted in Section 3. the aim is not to upgrade expressions $A \sqsupset B$ as much as possible to $A \supset B$, but to do so only for specific formulas that are implicative parts of Abs, viz. $\forall y(y \in\{x \mid A(x)\} \sqsupset A(y))$ and $\forall y(y \notin\{x \mid A(x)\} \sqsupset \neg A(y))$. Consider the formulas

$$
\mathrm{Q}(t \in\{x \mid A(x)\} \wedge \check{\neg} A(t)) \text { and } \mathrm{Q}(t \notin\{x \mid A(x)\} \wedge \check{\neg} \neg A(t))
$$

in which $t$ is a set theoretical term and, if it is a variable, $\mathrm{Q}$ is a quantifier over that variable. In view of Abs, the left formula PFS-entails $\mathrm{Q}(t \notin\{x \mid A(x)\} \wedge$ $t \in\{x \mid A(x)\} \wedge \neg A(t))$ and the right formula PFS-entails $\mathrm{Q}(t \in\{x \mid A(x)\} \wedge t \notin$ $\{x \mid A(x)\} \wedge \check{\neg} \neg A(t))$. So a bit of calculation shows that formulas of the forms in (3) may be safely taken as abnormalities. Proceeding thus, we obtain an adaptive logic that minimizes inconsistencies in view of abnormalities of the form $A \wedge \neg A$ and minimizes uncleanness in view of the abnormalities from (3).

The conclusion then is as follows. First, unless there are very special reasons to refrain from applying the defeasible rule in its full generality, the recipe leads to the following schema, considering multiple local premises (but still restricting to the lower limit CLuNs).

$$
\begin{array}{ll}
\mathrm{A}_{1} & \Delta_{1} \\
\vdots & \vdots \\
\mathrm{A}_{n} & \Delta_{n} \\
\hline \mathrm{B} & \Delta_{1} \cup \ldots \cup \Delta_{n} \cup\left\{\mathrm{A}_{1} \wedge \ldots \wedge \mathrm{A}_{n} \wedge * \mathrm{~B}\right\}
\end{array}
$$

Where $* \check{\neg} B=B$ and $* B=\check{\neg} B$ in case the first symbol in $B$ is not $\check{\neg}$. Call $\mathrm{A}_{1} \wedge \ldots \wedge \mathrm{A}_{n} \wedge * \mathrm{~B}$ the typical abnormality for the defeasible rule $\mathrm{A}_{1}, \ldots, \mathrm{A}_{n} / \mathrm{B}$. There is no reason to prefer abnormalities obtained by dropping one or more conjuncts of $A_{1} \wedge \ldots \wedge A_{n} \wedge * B$ because if the shorter formula can be considered as false, then so can the longer one. When one wants to introduce several 
defeasible rules, each of them may be given its typical abnormality. Two points of attention then are: (i) one should check which rules need to hold in their formal generality and which should be restricted to specific formulas - the AFS case illustrates this candidly - and (ii) one should study the effect of the typical abnormality of a rule on other rules in view of the logical and conceptual context - see the example discussed above.

The occurrence of the classical negation $\leftrightharpoons$ in the typical abnormality may look like causing trouble in paraconsistent contexts, especially for dialetheists. The matter will be discussed in Section 5 .

When the lower limit logic is not CLuNs, the typical abnormality is easily adjusted. Two adjustments may be required. (i) Sometimes restrictions on the subformulas of abnormalities need to be modified or removed. Thus the restriction $A \in \mathcal{F}^{a}$ needs sometimes to be replaced, for example by $A \in \mathcal{F}$, in which $\mathcal{F}$ is the set of (open and closed) formulas of standard predicative languages. (ii) Sometimes logical symbols are classical within the considered

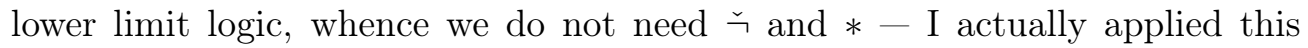
already within the present paper. I do not enter this any further as the matter is mainly technical.

Let us consider some typical abnormalities for other rules. I mentioned three defeasible rules of inductive generalization. In each of them $A, B$ and $C$ are disjunctions of literals and there are some further restrictions. The typical abnormalities for each of the rules can be read off below in somewhat simplified form - as the lower limit is $\mathbf{C L}$, the standard negation is classical:

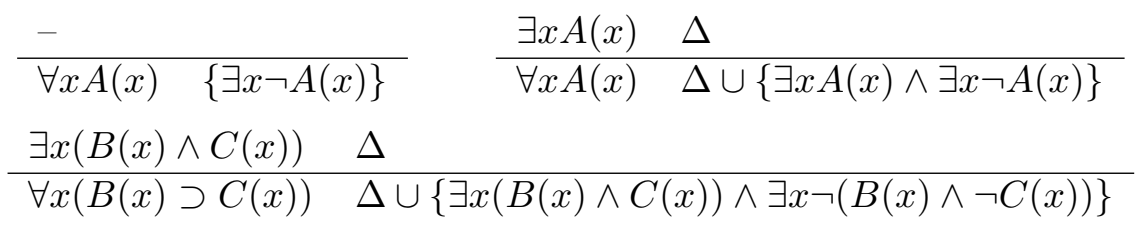

An inconsistency can be seen as a negation glut: that $v_{M}(A)=1$ justifies $v_{M}(\neg A)=0$ on the $\mathbf{C L}$-semantics, but actually $v_{M}(\neg A)=1$. A negation gap is where $v_{M}(A)=0$, which justifies $v_{M}(\neg A)=1$ on the CL-semantics, and nevertheless $v_{M}(\neg A)=0$. Along this line, one may consider gluts and gaps for every logical symbol of the standard predicative language. Adaptive logics minimizing gaps and gluts were studied [Batens, 2016]. Suppose that $\rightarrow$ is a glutty implication, whereas $\supset$ is the classical implication. A glutty implication is obviously not detachable, as there are models in which $v_{M}(A \rightarrow B)=1=$ $v_{M}(A)$ and $v_{M}(B)=0$. This brings us to something very close to (2). In order to minimize implication gluts, we want the following rule and abnormality:

$$
\begin{array}{ll}
A \rightarrow B \quad \Delta \\
\hline A \supset B \quad \Delta \cup\{(A \rightarrow B) \wedge A \wedge \neg B\}
\end{array}
$$


If glutty implications are combined with glutty negations (and possibly more oddities), the negation needs to be replaced by $\asymp$. All glutty and gappy logical symbols may be given defeasible rules to minimize them and the insight gained in this section will provide the rules with typical abnormalities.

\section{A Puzzle In Inconsistency-Adaptive Logics}

Classical negation occurs in the typical abnormalities from the previous section. Some will see this as problematic in paraconsistent contexts. Of course, if classical negation is not definable in a paraconsistent logic, one may add it, possibly forbidding its occurrence in premises and conclusion. Yet especially dialetheists will have objections to such move as they consider classical negation as a tonk-like operator. This conclusion is related to the dialetheist view that all true knowledge should form a single body, phrased within a single language and organized by The True Logic and that this body is necessarily inconsistent in view of the Liar paradox, paradoxes of set theory, etc. I shall not discuss the dialetheist position here, but rather argue that, for two reasons, the typical abnormalities do not lead to a situation that is at odds with dialetheism.

The first reason is that, due to the structural properties and functioning of negation, the typical abnormalities do not require that classical negation ever occurs either in them or elsewhere in a proof. First of all, look at two basic defeasible rules for negation: ${ }^{28}$

$$
\begin{array}{ll}
\neg A & \Delta \\
\hline \neg A & \Delta \cup\{A \wedge \neg A\}
\end{array}
$$$$
\begin{array}{ll}
A & \Delta \\
\hline \check{\neg} \neg A & \Delta \cup\{A \wedge \neg A\}
\end{array}
$$

However, once the adaptive logic is characterized in terms of the Standard Format, these rules need not be mentioned. Applications of the generic conditional rule RC may be phrased completely in the standard language, without ever writing a classical negation. Here are two examples, an application of Disjunctive Syllogism and one of Modus Tollens.

\begin{tabular}{ll}
$A \vee B$ & $\Delta$ \\
$\neg A$ & $\Theta$ \\
\hline$B$ & $\Delta \cup \Theta \cup\{A \wedge \neg A\}$
\end{tabular}

$$
\begin{array}{ll}
A \supset B & \Delta \\
\neg B & \Theta \\
\hline \neg A & \Delta \cup \Theta \cup\{B \wedge \neg B\}
\end{array}
$$

The classical negation in (5) signifies that, provided the abnormality introduced by the condition can be held to be false, $A$, respectively $\neg A$, can be considered as consistently false; spelled out, $\neg A$ signifies that $A$ is consistently

\footnotetext{
${ }^{28}$ Sometimes the $A$ in the abnormality is restricted, for example to atomic formulas, as is required when CLuNs is the lower limit logic. Sometimes several defeasible rules are required as in AFS.
} 


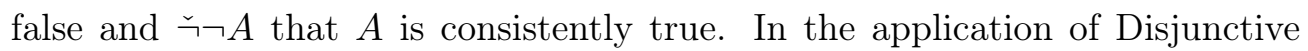
Syllogism, if the local premises are true and $A$ is consistent, then $B$ is bound to be true. That $A$ is consistent is nowhere stated. The fact that the conclusion line is unmarked indicates that (the members of $\Delta \cup \Theta$ as well as) $A \wedge \neg A$ can be held to be false, which means that $A$ is consistent and in that case $B$ is bound to be true if the local premises are true. ${ }^{29}$ The reasoning is similar for the application of Modus Tollens, except that here the consistency of $B$ matters. The situation is analogous for all applications of the generic conditional rule $\mathrm{RC}$ in $\mathbf{C L u N s}{ }^{m}$ and similar inconsistency-adaptive logics.

There is a second reason why the dialetheist should not eschew adaptive logics. The typical abnormality as defined in Section 4. works not only with classical negation, but works equally well with a paraconsistent negation. So where the symbol $\leftrightharpoons$ (defining $*$ ) in (4) is a negation that is paraconsistent and not also paracomplete, (4) still works fine: if all members of the condition can be held to be false, the conclusion follows from the premises.

This comment does not concern (5), the basic rule for negation. This rule, or rather both of them, are still unacceptable for the dialetheist because in it the symbol $\leftrightharpoons$ in the conclusion needs to be classical. As explained, however, there

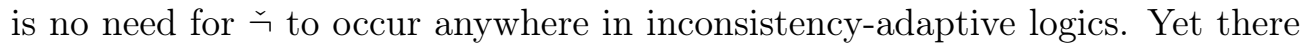
still is a catch. Suppose that the dialetheist position gets generally recognized, that the methodology of the sciences is spelled out in terms of, say, the LPnegation [Priest, 1987], and that scientists would actually apply LP rather than requiring, presupposing and sometimes pretending that their theories be consistent, then the dialetheist might phrase the whole scientific methodology in terms of adaptive logics based on LP. If the condition is false, the local conclusion will follow from the local premises. A hindrance for dialetheists will be that, in the preceding sentence, "false" needs to have the meaning with which I use it: consistently false, not false as meant by Graham Priest [Priest, 1987]. The latter meaning is that $A$ is false iff $\neg A$ is true; this entails that $A$ and $\neg A$ are both false in case they are both true, as may happen in paraconsistent contexts. The situation seems rather crucial. All instances of $A \wedge \neg A$ and all instances of $A \wedge \neg A \wedge \neg B$ are false in the sense of Priest. There is no point in asking whether they can be held to be false in view of the premises. They are false in Priest's sense, now, yesterday, tomorrow and always because their negation is true, even logically true: $\neg(A \wedge \neg A)$ is an LP-theorem; it is LP-equivalent to $\neg A \vee A$. From here on I return to my use of false.

Just for the record, a comment on two related negation-like entities. A paracomplete negation, according to which $A$ and its negation may be jointly false,

\footnotetext{
${ }^{29}$ Obviously, from $\neg A \vee B$ and $A$ follows $B$ on the condition $A \wedge \neg A$.
} 
is insufficient for adaptive logics to work decently. If the $\check{\neg}$ in (4) is paracomplete, the falsehood of the condition is insufficient for the local conclusion to follow from the local premises. The second negation-like entity is the arrowbottom construction, $A \rightarrow \perp$, in which $\rightarrow$ is a detachable implication and $\perp$ a bottom operator. ${ }^{30}$ This is obviously a kind of negation of $A$. Dialetheists have argued that $A \rightarrow \perp$, for $\rightarrow$ a relevant implication, ${ }^{31}$ allows them, just as much as the classical logician or intuitionist, to commit themselves to the falsehood of a certain statement $A$ in that $A \rightarrow \perp$ connects the truth of $A$ to triviality.

Some paragraphs ago, I argued that there is a problem for dialetheists to apply adaptive logics. Quite unexpectedly, however, there seems to be a way out. I am not a dialetheist, recently I even got doubts on the viability of dialetheism. Yet, these doubts are not related to what follows. Consider the following rules and their typical abnormalities:

$$
\begin{array}{lllll}
\neg A & \Delta & & A & \Delta \\
\cline { 1 - 2 } & \Delta \cup\{\neg A \wedge \neg(A \rightarrow \perp)\} & \neg A \rightarrow \perp & \Delta \cup\{A \wedge \neg(\neg A \rightarrow \perp)\}
\end{array}
$$

Dialetheists claim that true inconsistencies are exceptional. So, in nonexceptional situations, that $\neg A$ is given justifies one to defeasibly connect $A$ to triviality and that $A$ is given justifies one to defeasibly connect $\neg A$ to triviality.

The typical abnormality may look problematic, but it is not. For most relevant implications, $\neg(A \rightarrow B)$ is derivable from $A \wedge \neg B$. Where this is the case, $A \wedge \neg A$ is sufficient to derive both $\neg A \wedge \neg(A \rightarrow \perp)$ and $A \wedge \neg(\neg A \rightarrow \perp)$ because $\neg \perp$ is a theorem of $\mathbf{L P}$. To prevent readers from getting overoptimistic, let me point out that the 'negation' $\ominus$, defined by $\ominus A={ }_{d f} A \rightarrow \perp$, is a paracomplete negation. Clearly, $A \vee \ominus A$ is not a theorem unless the relevant $\rightarrow$ is downgraded to a detachable material implication.

It seems to me that the rules and abnormalities in (6) look extremely interesting from a dialetheist point of view. They allow dialetheists to express their commitment to the falsehood of a statement in the sense that the falsehood of $A$ connects the truth of $A$ to triviality. Moreover, they may do so without ever using classical negation - dialetheist may continue to catalogue that as a tonk-like operator. So (6) seems to provide a means for dialetheists to apply an inconsistency-adaptive logic without committing themselves to classical negation. Exploring the consequences of this insight obviously deserves a careful study, but that goes beyond the present paper. Moreover, adaptive

\footnotetext{
${ }^{30}$ A bottom operator is characterized by the rule "from $\perp$ to derive $A$ ".

${ }^{31}$ The intended relevant implications are not those from the well-known and very rich systems devised by Ackermann Ackermann, 1956, Church Church, 1951 and especially Anderson and Belnap Anderson, Belnap 1975 Anderson et al., 1992 but of much weaker systems surveyed by Routley [Routley, 1982] and Brady [Brady, 2006].
} 
consequences derived on a condition $\Delta$, remain to be justified in terms of the joint falsehood, in the dialetheist sense, of the members of $\Delta$.

\section{In Conclusion}

The problem I set out to solve concerned cases where one has an idea for devising an adaptive logic in terms of a defeasible rule. The easier case was where the set of abnormalities was given together with the lower limit logic for ampliative adaptive logics or together with the upper limit logic for corrective adaptive logics. If the idea for the adaptive logic comes in terms of a rule, the set of abnormalities has to be delineated. I presented an extremely simple and transparent recipe for doing so and argued that, except for very special cases, the recipe leads to an adequate result.

A further important point deserves to be mentioned. I have shown that there is a number of formerly unsolved difficulties for dialetheists who try to invoke inconsistency-adaptive logics. For me logics are instruments. Instruments may be independent of the philosophical and ideological views of those who use them. So it seems an important feature that adaptive logics as well as the proposed recipe work fine for dialetheists. Disagreements with dialetheists is not an excuse for hiding that, unlike what one might expect, adaptive logics turn out sensible and useful instruments for them.

A very different conclusion is not about generality but about specificity. There is a huge number of different adaptive logics of inductive generalization. This is not only required because of the many disagreements between philosophers of science on inductive methods. It is also necessary in view of the very different domains of application. To give just one example, the non-logical terms of one language may be well entrenched technical terms and those of another language may be taken straight from natural language. Further differences will depend on the underlying conceptual framework, on the presence of articulated observational criteria, and so on. Such differences may have an effect on the suitability of a specific inductive method. The situation for other ampliative adaptive logics is analogous.

Similar comments apply to corrective adaptive logics. The upper limit logic is known beforehand, but there are many ways to approach it: different strategies, different lower limit logics, and for each combination of a strategy and lower limit logic, different sets of abnormalities. Of course, not every specific circumstance determines a single adaptive logic. Nevertheless, the choice of a suitable adaptive logic will be largely determined by properties of the theory or domain to which it is applied. Mathematical theories have generally conceptual structures that are much simpler that most empirical theories. So they usually require a stronger lower limit logic, validating full Replacement 
of Identicals and reducing all statements to truth-functions of literals - truthfunctions in the broad sense including quantifiers. But apart from such rough classifications, both mathematical and empirical theories will require careful analysis in order to select the specific non-logical axioms in view of the lower limit logic. Adaptive mathematical theories [Batens, 2014 Batens, 2019] are a case in point.

Part of the importance of the present paper and of the recipe is related to insights that have grown over the years. In the early days, adaptive logics seemed to present an attractive approach to handle certain problems. Examples were (i) inconsistencies coming up unexpectedly in a theory that was intended as consistent or (ii) devising a precise formulation of a given method. By and large, the impression was that adaptive logics were very general tools that could be efficiently applied in nearly all circumstances. Only over the years did it become clear that especially the choice of corrective adaptive logics depends heavily on the context. When a problem is located, adaptive logics do not provide one with a tool that in itself warrants success. One has to carefully choose a language in which to formulate the problem. One has to carefully select the way in which the theory or the data, in which the problem arises, are phrased. Recently, especially with the application to Fregean set theories (sic), it turned out that sometimes one even has to tailor the adaptive logic in view of its application. On the one hand, this shows to what extend Dudley Shapere was right in propagating content guidance and learning how to learn Shapere, 2004. On the other hand, it made necessary the search for the present recipe: content guidance provokes more frequently the need for adaptive logics devised in view of defeasible rules.

Acknowledgements. I want to thank the referee and the editor for their careful work.

\section{References}

Ackermann, 1956 - Ackermann, W. "Begründung einer strengen Implikation", Journal of Symbolic Logic, 1956, Vol. 21, pp. 113-128.

Anderson, Belnap 1975 - Anderson, A.R., Belnap, N.D. Entailment. The Logic of Relevance and Necessity, Vol. 1. Princeton University Press, 1975.

Anderson et al., 1992 - Anderson, A.R., Belnap, N.D., Dunn, J.M. Entailment. The Logic of Relevance and Necessity, Vol. 2. Princeton University Press, 1992.

Batens, 2019 - Batens, D. "Adaptive Fregean set theory", Studia Logica, 2019. https: //doi.org/10.1007/s11225-019-09882-1

Batens, 1985 - Batens, D. "Dynamic dialectical logics as a tool to deal with and partly eliminate unexpected inconsistencies", in: The Logic of Discovery and the Logic of Discourse, J. Hintikka, F. Vandamme (eds.), Plenum Press, New York, 1985, pp. 263-271. 
Batens, 1986 - Batens, D. "Dialectical dynamics within formal logics", Logique et Analyse, 1986, Vol. 114, pp. 161-173.

Batens, 1989 - Batens, D. "Dynamic dialectical logics", in: Paraconsistent Logic. Essays on the Inconsistent, G. Priest, R. Routley, J. Norman (eds.), Philosophia Verlag, München, 1989, pp. 187-217.

Batens, 2000 - Batens, D. "Minimally abnormal models in some adaptive logics", Synthese, 2000, Vol. 125, pp. 5-18.

Batens, 2002 - Batens, D. "On some remarkable relations between paraconsistent logics, modal logics, and ambiguity logics", in: Paraconsistency. The Logical Way to the Inconsistent, W.A. Carnielli, M.E. Coniglio, and I.M.L. D'Ottaviano (eds.), Marcel Dekker, New York, 2002, pp. 275-293.

Batens, 2004 - Batens, D. "The basic inductive schema, inductive truisms, and the research-guiding capacities of the logic of inductive generalization", Logique et Analyse, 2004, Vol. 185-188, pp. 53-84. (Appeared 2005)

Batens, 2005 - Batens, D. "On a logic of induction", in: Confirmation, Empirical Progress, and Truth Approximation. Essays in Debate with Theo Kuipers. Vol. 1, volume 83 of Poznan Studies in the Philosophy of the Sciences and the Humanities, R. Festa, A. Aliseda, J. Peijnenburg (eds.). Rodopi, Amsterdam/New York, 2005, pp. 221-242. (Contains uncorrected proofs; see [Batens, 2006 for correct version.)

Batens, 2005 - Batens, D. "The theory of the process of explanation generalized to include the inconsistent case", Synthese, 2005, Vol. 143, pp. 63-88. doi:10.1007/s11229-005-3114-3.

Batens, 2006 - Batens, D. "On a logic of induction", LEPS - Logic \& Philosophy of Science, 2006, Vol. IV, No. 1, pp. 3-32. (Corrected version of Batens, 2005].)

Batens, 2007 - Batens, D. "A universal logic approach to adaptive logics", Logica Universalis, 2007, Vol. 1, pp. 221-242.

Batens, 2009 - Batens, D. "Towards a dialogic interpretation of dynamic proofs", in: Dialogues, Logics and Other Strange Things. Essays in Honour of Shahid Rahman, C. Dégremont, L. Keiff, H. Rückert (eds.). College Publications, London, 2009, pp. $27-51$.

Batens, 2011 - Batens, D. "Logics for qualitative inductive generalization", Studia Logica, 2011, Vol. 97, No. 1, pp. 61-80. doi:10.1007/s11225-010-9297-8.

Batens, 2014 - Batens, D. "The consistency of Peano Arithmetic. A defeasible perspective", in: Modestly Radical or Radically Modest. Festschrift for Jean Paul van Bendegem on the Occasion of His 60th Birthday, P. Allo, B. Van Kerkhove (eds.), Vol. 24 of Tributes, College Publications, London, 2014, pp. 11-59.

Batens, 2015 - Batens, D. "Tutorial on inconsistency-adaptive logics", in: New Directions in Paraconsistent Logic, J.-Y. Béziau, M. Chakraborty, S. Dutta (eds.), Vol. 152 of Springer Proceedings in Mathematics $\&$ Statistics, Springer, 2015, pp. 3-38.

Batens, 2016 - Baten, D. "Spoiled for choice?", Journal of Logic and Computation, 2016, Vol. 26(1) pp. 65-95. E-published 2013: doi:10.1093/logcom/ext019. 
Batens, 2017 - Batens, D. "Abduction logics illustrating pitfalls of defeasible methods", in: Applications of formal philosophy: the road less travelled, R. Urbaniak, G. Payette (eds.), Vol. 14 of Logic, argumentation \& reasoning, Springer, Berlin, 2017, pp. 169-193.

Batens, Clercq, 2004 - Batens, D., De Clercq, K. "A rich paraconsistent extension of full positive logic", Logique et Analyse, 2004, Vol. 185-188, pp. 227-257. (Appeared 2005)

Batens et al., 2009 - Batens, D., De Clercq, K., Verdée, P., Meheus, J. "Yes fellows, most human reasoning is complex", Synthese, 2009, Vol. 166, pp. 113-131.

Batens, Haesaert, 2003 - Batens, D., Haesaert, L. "On classical adaptive logics of induction, Logique et Analyse, 2001, Vol. 173-175, pp. 255-290. (Appeared 2003)

Batens, Meheus, 2000 - Batens, D., Meheus, J. "The adaptive logic of compatibility", Studia Logica, 2000, Vol. 66, pp. 327-348.

Batens, Meheus, 2001 - Batens, D., Meheus, J. "On the logic and pragmatics of the process of explanation", in: Explanatory Connections. Electronic Essays Dedicated to Matti Sintonen, M. Kiikeri, P. Ylikoski (eds.), 2001, pp. 22.

Beirlaen, Aliseda, 2014 - Beirlaen, M., Aliseda, A. "A conditional logic for abduction", Synthese, 2014, Vol. 191, pp. 3733-3758.

Beirlaen, Straßer, 2013a - Beirlaen, M., Straßer, C. "Non-monotonic reasoning with normative conflicts in multi-agent deontic logic", Journal of Logic and Computation, 2013, Vol. 24, No. 6, pp. 1179-1207.

Beirlaen, Straßer, 2013b - Beirlaen, M., Straßer, C. "Two adaptive logics of normpropositions", Journal of Applied Logic, 2013, Vol. 11, No. 2, pp. 147-168.

Brady, 2006 - Brady, R. Universal Logic. CSLI Publications, Stanford, Cal., 2006.

Church, 1951 - Church, A. "The weak theory of implication", in: Kontrolliertes Denken. Untersuchungen zum Logikkalkül und zur Logik der Einzelwissenschaften, A. Menne, A. Wilhemy, H. Angsil (eds.), Vol. 16, Karl Alber, München, 1951, pp. $22-37$.

De Clercq, Verhoeven, 2004 - De Clercq, K., Verhoeven, L. "Sieving out relevant and efficient questions", Logique et Analyse, 2004, Vol. 185-188, pp. 53-84. (Appeared 2005)

Gauderis, Van De Putte, 2012 - Gauderis, T., Van De Putte, F. "Abduction of generalizations", Theoria, 2012, pp. 345-363.

Goble, 2014 - Goble, L. "Deontic logic (adapted) for normative conflicts", Logic Journal of the IGPL, 2014, Vol. 22, No. 2, pp. 206-235.

Horsten, Welch, 2007 - Horsten, L., Welch, P. "The undecidability of propositional adaptive logic", Synthese, 2007, Vol. 158, pp. 41-60.

Lycke, 2012 - Lycke, H. "A formal explication of the search for explanations: the adaptive logics approach to abductive reasoning", Logic Journal of the IGPL, 2012, Vol. 20, pp. 497-516.

Meheus, 2001 - Meheus, J. "Adaptive logics for question", Logique et Analyse, 2001, Vol. 173-175, pp. 135-164. (Appeared 2003) 
Meheus, 2003 - Meheus, J. "Paraconsistent compatibility", Logique et Analyse, 2003, Vol. 183-184, pp. 251-287. (Appeared 2005)

Meheus, 2004 - Meheus, J. "Adaptive logics and the integration of induction and deduction", in: Induction and Deduction in the Sciences, ed. by F. Stadler, Kluwer, Dordrecht, 2004, pp. 93-120.

Meheus, 2007 - Meheus, J. "Adaptive logics for abduction and the explication of explanation-seeking processes", in: Abduction and the Process of Scientific Discovery, O. Pombo, A. Gerner (eds.), Centro de Filosofia das Ciências da Universidade de Lisboa, Lisboa, 2007, pp. 97-119.

Meheus, 2011 - Meheus, J. "A formal logic for the abduction of singular hypotheses", in: Explanation, Prediction, and Confirmation, D. Dieks, W. J. Gonzalez, S. Hartmann, T. Uebel, M. Weber (eds.), Springer, Dordrecht, 2011, pp. 93-108.

Meheus, Batens, 2006 - Meheus, J., Batens, D. "A formal logic for abductive reasoning", Logic Journal of the IGPL, 2006, Vol. 14, pp. 221-236.

Meheus et al., 2010a - Meheus, J., Beirlaen, M., Putte, F.V.D. "Avoiding deontic explosion by contextually restricting aggregation", in: Deontic Logic in Computer Science, G. Governatori, G. Sartor (eds.), Springer, Dordrecht, 2010, pp. 148-165.

Meheus et al., 2010b - Meheus, J., Beirlaen, M., Putte, F.V.D., Straßer, C. "Nonadjunctive deontic logics that validate aggregation as much as possible", 2010, https://www.clps.ugent.be/sites/default/files/publications/nadl. pdf, accessedon09.04.2020.

Meheus, Provijn, 2004 - Meheus, J., Provijn, D. "Direct dynamic proofs for classical compatibility", Logique et Analyse, 2004, Vol. 185-188, pp. 305-317. (Appeared 2005)

Meheus et al., 2002 - Meheus, J., Verhoeven, L., Van Dyck, M., Provijn, D. "Ampliative adaptive logics and the foundation of logic-based approaches to abduction", in: Logical and Computational Aspects of Model-Based Reasoning, L. Magnani, N.J. Nersessian, C. Pizzi (eds.), Kluwer, Dordrecht, 2002, pp. 39-71.

Odintsov, Speranski, 2012 - Odintsov, S.P., Speranski, S.O. "On algorithmic properties of propositional inconsistency-adaptive logics", Logic and Logical Philosophy, 2012, Vol. 21, pp. 209-228.

Odintsov, Speranski, 2013 - Odintsov, S.P., Speranski, S.O. "Computability issues for adaptive logics in multi-consequence standard format", Studia Logica, 2013, Vol. 101, No. 6, pp. 1237-1262. doi:10.1007/s11225-013-9531-2.

Pogorzelski, Prucnal, 1975 - Pogorzelski, W.A., Prucnal, T. "The substitution rule for predicate letters in the first-order predicate calculus", Reports on Mathematical Logic, 1975, Vol. 5, pp. 77-90.

Priest, 1987 - Priest, G. In Contradiction. A Study of the Transconsistent. Oxford University Press, Oxford, 2006. Second expanded edition (first edition 1987).

Routley, 1982 - Routley, R. Relevant Logics and their Rivals, Vol. 1, Ridgeview, Atascadero, Ca., 1982. 
Shapere, 2004 - Shapere, D. "Logic and the philosophical interpretation of science", in: Alternative Logics. Do sciences need them?, ed. by P. Weingartner, Springer, Berlin, Heidelberg, 2004, pp. 41-54.

Straßer, 2010 - Straßer, C. "An adaptive logic framework for conditional obligations and deontic dilemmas", Logic and Logical Philosophy, 2010, Vol. 19, No. 1-2, pp. $95-128$.

Straßer et al., 2012 - Straßer, C., Meheus, J., Beirlaen, M. "Tolerating deontic conflicts by adaptively restricting inheritance", Logique et Analyse, 2012, Vol. 219, pp. 477-506.

Van De Putte et al., in press - Van De Putte, F., Beirlaen, M., Meheus, J. "Adaptive deontic logics", in: Handbook of Deontic Logic and Normative Systems, Vol. 2, D. Gabbay, J. Horty, X. Parent, R. van der Meyden, L. van der Torre (eds.), College Publications, in press.

Van De Putte, Straßer, 2012 - Van De Putte, F., Straßer, C. "A logic for prioritized normative reasoning", Journal of Logic and Computation, 2012, Vol. 23, pp. 563583. doi:10.1093/logcom/exs008.

Vanackere, 1999a - Vanackere, G. "Ambiguity-adaptive logic", Logique et Analyse, 1997, Vol. 159, pp. 261-280. (Appeared 1999)

Vanackere, 1999b - Vanackere, G. "Minimizing ambiguity and paraconsistency", Logique et Analyse, 1999, Vol. 165-166, pp. 139-160. (Appeared 2002)

Vanackere, 2000 - Vanackere, G. "HL2. An inconsistency-adaptive and inconsistencyresolving logic for general statements that might have exceptions", Journal of Applied Non-Classical Logics, 2000, Vol. 10, pp. 317-338.

Vanackere, 2001 - Vanackere, G. "The role of ambiguities in the construction of collective theories", Logique et Analyse, 2001, Vol. 173-175, pp. 189-214. (Appeared 2003)

Verdée, 2009 - Verdée, P. "Adaptive logics using the minimal abnormality strategy are $\Pi_{1}^{1}$-complex", Synthese, 2009, Vol. 167, pp. 93-104.

Verdée, Batens, 2016 - Verdée, P., Batens, D. "Nice embedding in classical logic", Studia Logica, 2016, Vol. 104, pp. 47-78. E-published since 2015: doi:10.1007/s11225015-9622-3.

Weber, De Clercq, 2002 - Weber, E., De Clercq, K. "Why the logic of explanation is inconsistency-adaptive", in: Inconsistency in Science, ed. by J. Meheus, Kluwer, Dordrecht, 2002, pp. 165-184.

Weber, Van Dyck, 2001 - Weber, E., Van Dyck, M. "Adaptive logic and covering law explanations", Logique et Analyse, 2001, Vol. 173-175, pp. 237-254. (Appeared 2003) 